\title{
Performance Evaluation of IPv4 and IPv6 over MPLS using OPNET
}

\author{
Suhail Ahmad \\ Computer Science \& \\ Engineering Department, \\ University of Kashmir, \\ Srinagar (J\&K), India
}

190006

\author{
Wajid Ali Hamdani \\ Computer Science \& \\ Engineering Department, \\ University of Kashmir, \\ Srinagar (J\&K), India
}

190006

\author{
Mohsin Hassan Magray \\ Computer Science \& \\ Engineering Department, \\ University of Kashmir, \\ Srinagar (J\&K), India
}

190006

\begin{abstract}
Over the last two decades, we have witnessed a rapid deployment of real-time applications on the Internet and many research works focus on Quality of Service (QoS), in particular using IPv4 (Internet Protocol Version 4). The inevitable exhaustion of the remaining IPv4 address pool has become progressively evident and as a result the evolution of Internet Protocol (IP) continues leading to the deployment of IPv6 QoS. Today, there is limited experience in the deployment of QoS for IPv6 traffic in MPLS backbone networks in conjunction with DiffServ (Differentiated Services) support. DiffServ itself does not have the ability to control the traffic flow whereas MPLS Traffic Engineering (TE) can control the traffic and can set up end-to-end routing path before data can be forwarded. Multi-Protocol Label Switching (MPLS) plays a key role in next generation networks by delivering QoS and traffic engineering features which is helpful in managing traffic when some links or paths are under and/or over utilized This paper presents a QoS performance study of some applications such as voice, video conferencing, mail and web over DiffServ with MPLS in IPv4/IPv6 networks using Optimized Network Engineering Tool (OPNET). The effectiveness of DiffServ and MPLS integration in IPv4/IPv6 network is illustrated and analyzed.
\end{abstract}

Keywords

MPLS, IPv4, IPv6, QoS, OPNET, OSPF.

\section{INTRODUCTION}

With the advances in information and communication technology (ICT) over the past few years, the computer networking is becoming the corner stone of modern society. But at the same time, service providers need to look at architectures that give them better monitoring and control of the network traffic so that the performance is optimal and there is minimal increase in network resources [1]. Mostly used protocol that serves the end users is the Internet Protocol version 4 (IPv4) addressing scheme. However, with the tremendous growth of the internet, the addresses provided by IPv4 have proven to be insufficient and inadequate which has lead to the evolution of IPv6 [2].

The performance of the network and parts of networks can be affected by numerous parameters which result in an impact on the QoS parameters such as jitter, datagram or packet loss, latency, poor transfer rates and bandwidth quality. Numbers of approaches are available which can be used to improve network performance. In this paper, we have analyzed the performance of two virtual core network environments:

a. MPLS over core IPv4 network.

b. MPLS over core IPv6 network.
This paper is organized as follows: section 2 presents the survey of related work in this area. In section 3 we present an overview of MPLS. Section 4 gives the details of simulation environment. Section 5 discusses the results obtained gives the analysis of results. Finally conclusion is given in Section 6.

\section{RELATED WORK}

In [1], Rozita Yunos et al studies the performance evaluation between IPv4 and IPv6 with Linux MPLS tunnel. In their investigation they have concluded that the tunneling provide the means for the coexistence of IPv4 and IPv6 populations at the expense of the TCP transmission performance. In [2], the authors evaluate IPv4 to IPv6 transition performance and the parameters they have studied are throughput, latency, host utilization and TCP connection time. There is a very large literature on general aspects of performance issues of implementing MPLS over IPv4 and IPv6 networks as described in $[6,7,8]$. In [6], M. K. Powel et al made a comparative analysis of MPLS over Non-MPLS networks and shows MPLS have a better performance over traditional IP networks. Simulations on QualNet 4.0 simulator of MPLS and NonMPLS networks are compared by considering the parameters such as packet loss, throughput and end-to-end delay on the network traffic. In [7], the authors have focused on the analytical models to measure efficiency of voice over IP network with applications on MPLS network. In their analysis, network models are presented to support quality of service (QoS) requirements and traffic engineering standards supported by MPLS. In [8], M. T. Aziz et al have compared the packet delay variation of VoIP in IPv4 and IPv6 over MPLS.

\section{MPLS OVERVIEW}

Multi Protocol Label Switching (MPLS) is architecture for fast packet switching and routing. It provides the designation, routing, forwarding and switching of traffic flows through the network. MPLS has been proposed as a solution to overcome some limitations, drawbacks and problems associated with the network model that is currently used in the core network nowadays [3,4]. All packets are labelled before being forwarded and consequently, at down-stream routers, analysis of the packet's network layer header is not required [5]. Although the original idea behind the development of MPLS was to facilitate fast packet switching, currently its main goal is to support traffic engineering and provide quality of service (QoS).

\subsection{Traffic Engineering in MPLS Networks}

Traffic Engineering (TE) is a mechanism put in place to control the flow of traffic in networks and it provides the performance optimization of the network resources. The main characteristics of TE are fault-tolerance, optimum resource utilisation and resource reservation [11]. The basic objective of the 
consideration of TE is to improve quality of service of some applications and use the available network resources efficiently. There are some important factors, which are needed for TE. These factors are; Path Selection, Traffic Management, Direction of Traffic along Computed Paths and Distribution of Topology Information.

The LSPs in the MPLS network are established and the labels are distributed on each of the hops along the LSPs before packets could be forwarded. The LSPs can be established either by explicitly routed LSP or control driven LSP. Control driven LSPs can also be referred to as hop-by-hop LSP and are set by the use of LDP protocol. Explicitly routed LSPs can also be referred to as constraint based LSPs (CR-LSPs), which are specified in the setup message. At each hop, a label request is sent to the next hop along the LSP [12].

There are basically two protocols used to set CR-LSPs in MPLS. These protocols are; Resource Reservation Protocol (RSVP) and Constraint based routed LDP (CR-LDP).

\subsection{MPLS Architecture}

The MPLS domain is described as "a contiguous set of nodes which operate MPLS routing and forwarding". MPLS domain is divided into MPLS core which consists of Label Switch Routers (LSRs) and MPLS edge which consists of Label Edge Routers (LERs). The main terminologies of MPLS technology are explained as follows:

\section{Label Switch Router (LSR)}

Any router which is located in the MPLS domain and forwards the packets based on label switching is called LSR. When an LSR receives a packet it checks the look-up table and determines the next hop, before forwarding the packet to next hop it removes the old label from the header and attaches new label.

\section{Label Edge Router (LER)}

A packet enters into MPLS domain through LER which is called Ingress router. Packet leaves the MPLS domain through LER which is called Egress router. LER has an ability to handle L3 lookups and is responsible for adding or removing the labels from the packets as they enter or leave the MPLS

\section{Label Distribution Protocol (LDP)}

It is a protocol in which the label mapping information is exchanged between LSRs. .It is responsible in establishing and maintaining labels.

\section{Forward Equivalence Class (FEC)}

It is considered as the set of packets which have related characteristics and are forwarded with the same priority in the same path. This set of packets is bounded to the same MPLS label. Each packet in MPLS network is assigned with FEC only once at the Ingress router.

\section{Label Switched path (LSP)}

LSP is the path set by the signalling protocols in MPLS domain. In MPLS domain there exists number of LSPs that originate at Ingress router and traverses one or more core LSRs and terminates at Egress router.

\section{SIMULATION ENVIRONMENT}

OPNET simulator was used to compare MPLS over core IPV4 network with MPLS over core IPV6 network. OPNET is a realtime simulator designed mainly for the design and analysis of network models. The network topology used to carry out this research consists of 8 routers, out of these 8 routers 3 routers are LER and 5 routers are LSR. Besides these routers 4 applications have been simulated over these networks namely voice, video, database and ftp. 2 servers are used one for ftp and another for database. The links used for connection are PPP_DS1 links. MPLS has been implemented over the network. WFQ_DSCP class based a QoS technique has been implemented. In addition to all this background traffic is running in the network. Figure 1 below shows the IPv4 over MPLS network for this research and the same network is used for IPv6 over MPLS simulation as shown in Figure 2. In IPv4 over MPLS network OSPF routing protocol is used and in IPv6 over MPLS network OSPFv3 routing protocol is used. Four FECs are configured each for video, voice, database and ftp applications, also traffic trunks are configured for each running applications. Two LSPs are configured, one for video-voice LSP and another for database-ftp LSP. Table 1 gives an overview of the applications being run in this simulation. domain.

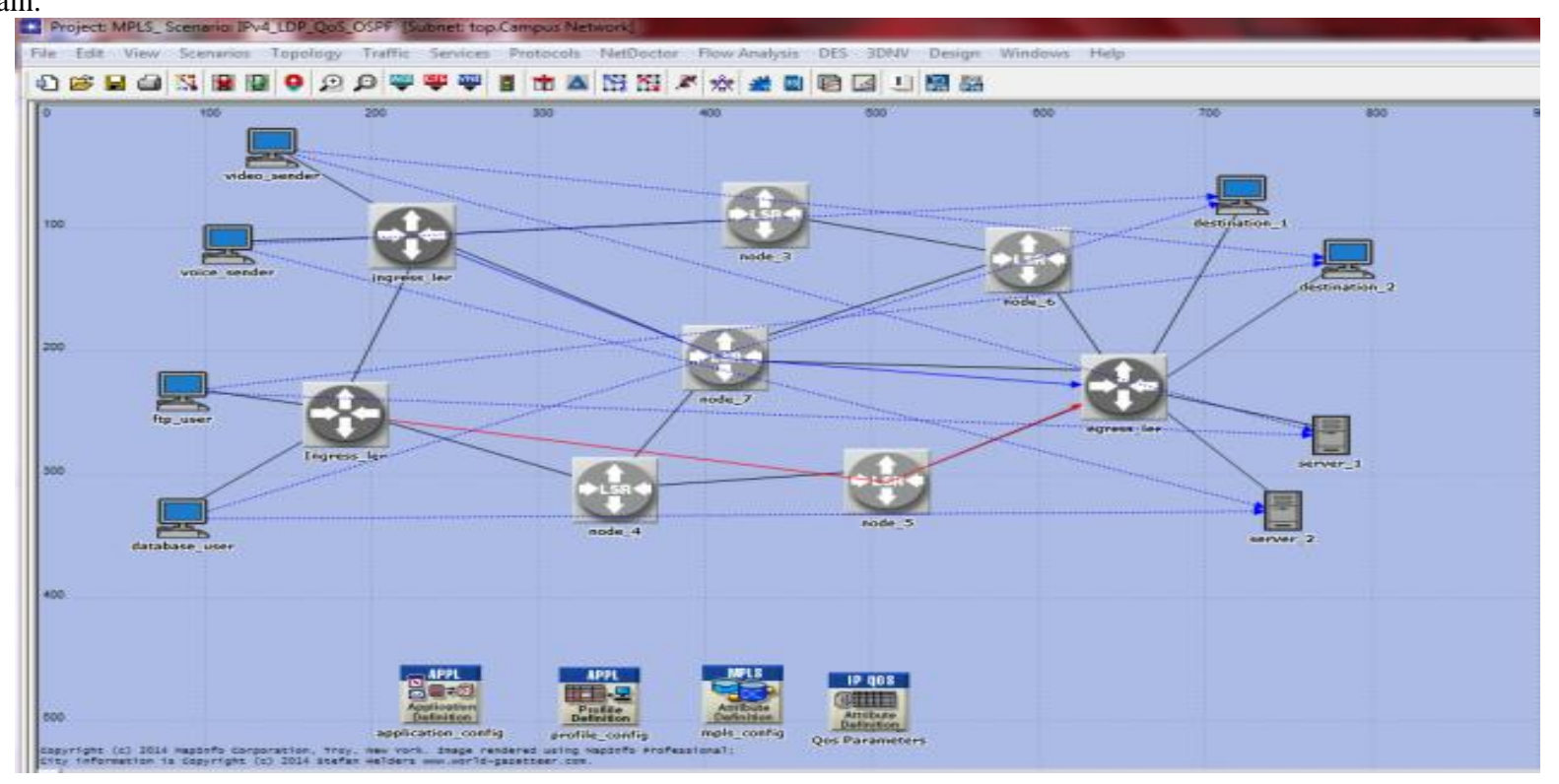

Fig.1: IPv4 over MPLS 


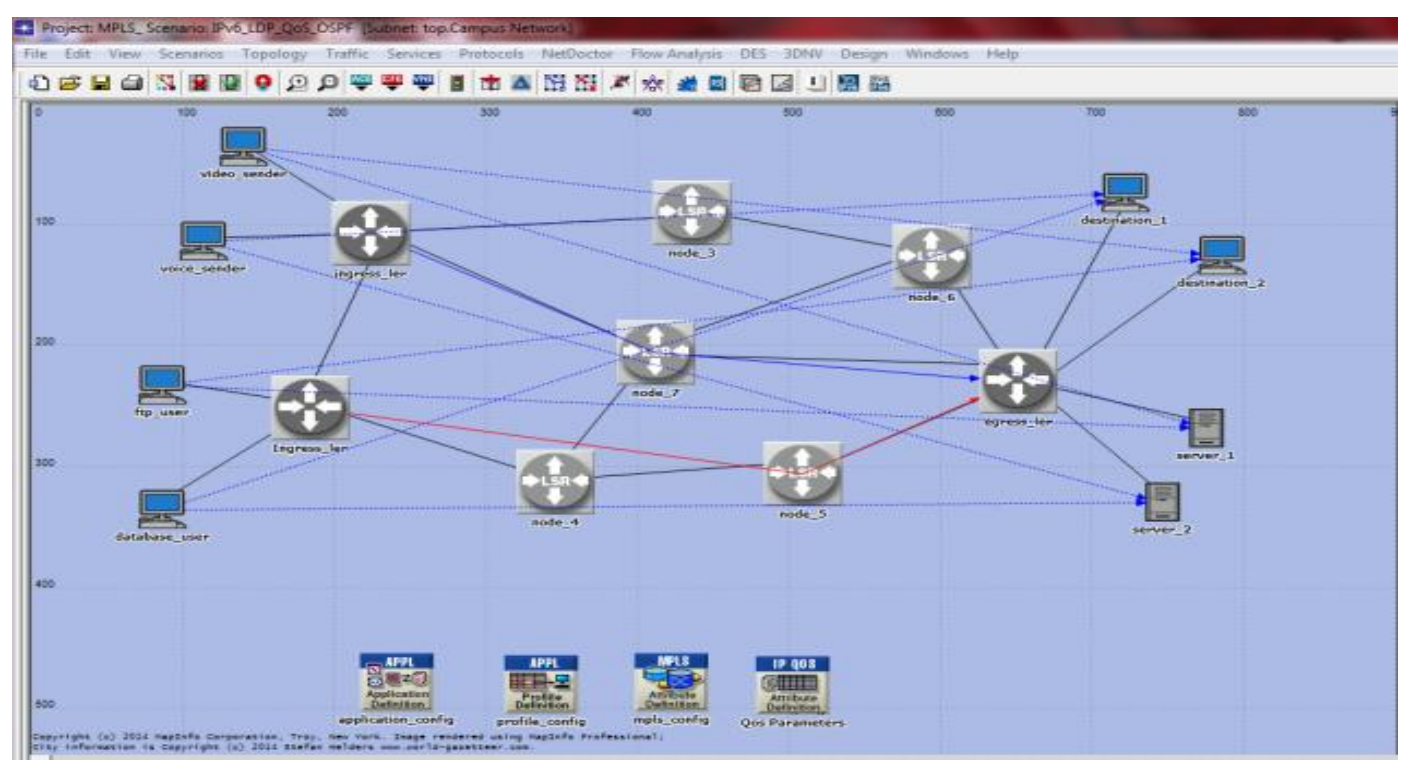

Fig.2: IPv6 over MPLS

Table 1: Selected Applications and their use.

\begin{tabular}{|l|l|}
\hline Application & Situations where used \\
\hline $\begin{array}{l}\text { FTP (High } \\
\text { Load) }\end{array}$ & $\begin{array}{l}\text { File servers e.g. Rapidshare and } \\
\text { Ubuntu mirror servers }\end{array}$ \\
\hline $\begin{array}{l}\text { Voice (GSM } \\
\text { Quality) }\end{array}$ & VOIP Applications e.g. Skype \\
\hline $\begin{array}{l}\text { Video } \\
\text { Conferencing } \\
\text { (High Resolution } \\
\text { Video) }\end{array}$ & $\begin{array}{l}\text { Remote news reporting and } \\
\text { interviews e.g. CNN and BBC }\end{array}$ \\
\hline $\begin{array}{l}\text { Database (High } \\
\text { Load) }\end{array}$ & For Data Storage \\
\hline
\end{tabular}

\section{SIMULATION RESULTS}

The various parameters evaluated are as follows:

Throughput: It is clear from figure 3 that the throughput for IPv6 over MPLS network increase throughout the simulation period and was $35553.04 \mathrm{bits} / \mathrm{sec}$ at the end of the simulation period. It can also be seen that the throughput for the IPv4 over MPLS network increases throughout the simulation period and was $28442.08 \mathrm{bits} / \mathrm{sec}$ at the end of the simulation.

The reason for the difference in throughput is in IPv4 each packet must be processed and checksum is computed, and each route that processes the packet must process the optional field, while in IPv6 the optional fields as well as non essential fields to extension headers are placed only after IPv6 header. This results in fast routing. Besides, we used traffic engineering to reduce the packet drop hence keeping the packets in network.

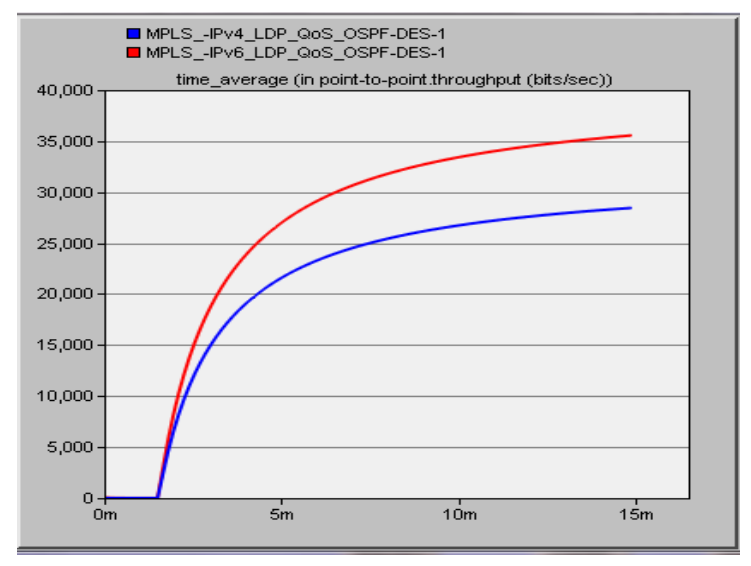

Fig.3: Throughput for IPV4 and IPV6 over MPLS

Link Utilization: From Figure 4, it can be seen that the link utilization for IPv6 over MPLS network increase throughout the simulation period and was 2.302658 at the end of the simulation period. It can also be seen that the throughput for the IPv4 over MPLS network increases throughout the simulation period and was 1.842104 at the end of the simulation.

Increased in throughput in IPv6 compared to IPv4 depicts that there are more packets in the network which are being routed which leads to more utilization of links in IPv6 than in IPv4. Besides, MPLS traffic trunks have been implemented which plays a wide role in increasing the utilization. 


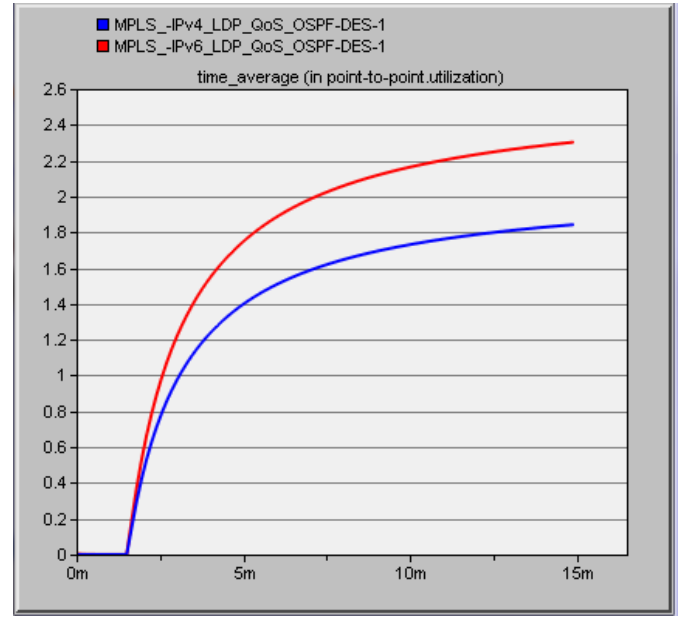

Fig.4: Link Utilization

End to end Delay: From Figure 5 below, it can be seen that the End to end delay for IPv6 over MPLS network increase throughout the simulation period and was $6.152128 \mathrm{sec}$ at the end of the simulation period. It can also be seen that the End to end delay for the IPv4 over MPLS network increases throughout the simulation period and was $5.641169 \mathrm{sec}$ at the end of the simulation.

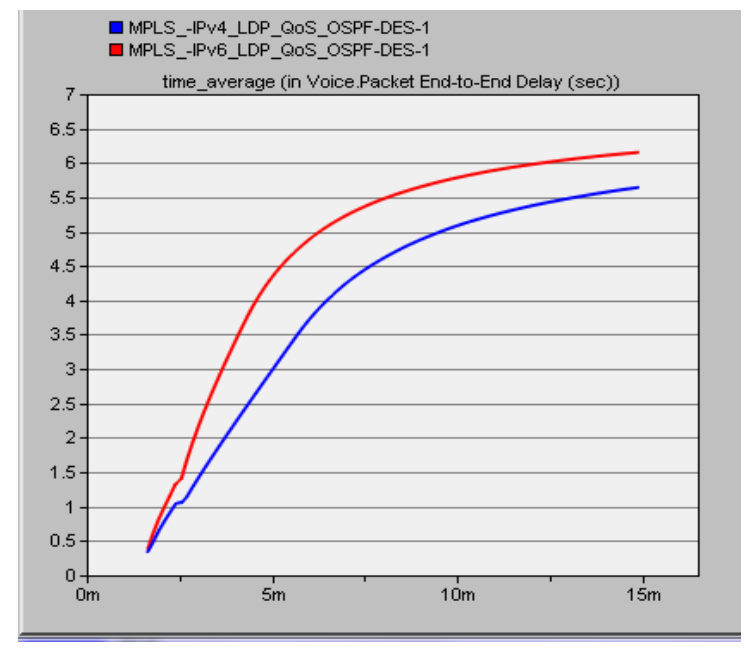

Fig.5: End to end Delay

Since IPv6 has a bigger packet size also header size is larger than that of IPv4 therefore the end-to-end delay is more in IPv6 than that of IPv4. In MPLS network the different type of traffic is sent over the different routes using traffic engineering, hence decreasing the delay very considerably. Providing trunks and QoS has reduced the delay in MPLS.

Voice Jitter: As depicted in figure 6, it can be seen that the voice jitter for IPv6 over MPLS network is maximum at time nearly around $2 \mathrm{~min}$, the value for voice jitter is $0.001499 \mathrm{sec}$ it goes on decreasing until it was 0.000135 at the completion of simulation. The voice jitter for the IPv4 over MPLS network was $0.001003 \mathrm{sec}$ until the 2nd minute of the simulation when it started to rapidly decrease until it was $0.000146 \mathrm{sec}$ at the end of the simulation.

Jitter is the rate of change of inter packet delay. In IPv6 over MPLS network since delay is higher than that of IPv4 over MPLS network; therefore jitter is also higher in IPv6 over MPLS network. The result collected for different parameters are shown in table 2 .

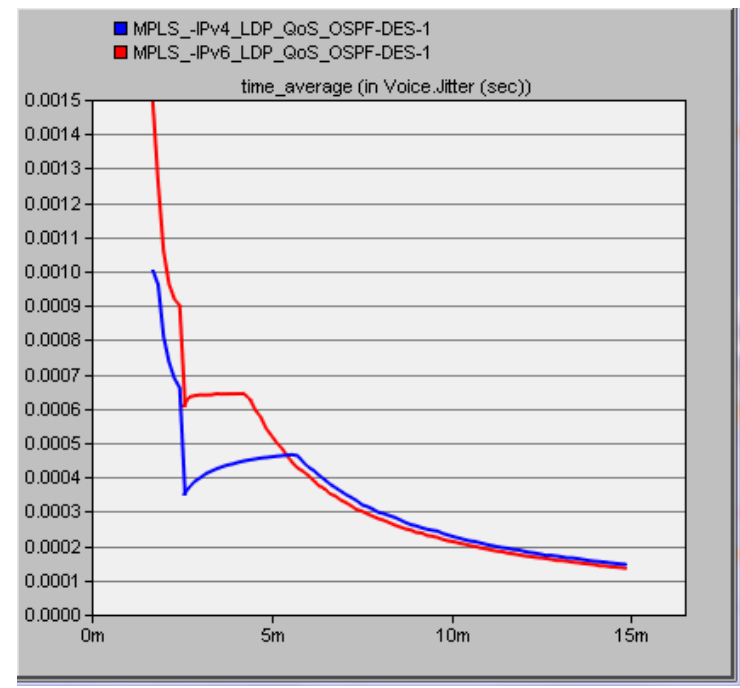

Fig.6: Voice Jitter

\begin{tabular}{|l|l|l|l|l|}
\hline \multicolumn{2}{|c|}{ Attributes } & Maximum & Minimum & Average \\
\hline $\begin{array}{l}\text { Throughpu } \\
\text { t } \\
\text { (bits/sec) }\end{array}$ & IPv4 & 28442.08 & 0 & 20768.02 \\
\cline { 2 - 5 } & IPv6 & 35552.15 & 5.090909 & 25962.72 \\
\hline $\begin{array}{l}\text { Link } \\
\text { Utilization }\end{array}$ & IPv4 & 1.842104 & 0 & 1.345079 \\
\cline { 2 - 5 } & IPv6 & 2.302658 & 0.00333 & 1.681523 \\
\hline $\begin{array}{l}\text { End to end } \\
\text { Delay } \\
\text { (sec) }\end{array}$ & IPv4 & 5.641169 & 0.357446 & 4.040027 \\
\cline { 2 - 5 } & IPv6 & 6.152128 & 0.397252 & 4.82321 \\
\hline $\begin{array}{l}\text { Voice } \\
\text { Jitter } \\
\text { (sec) }\end{array}$ & IPv4 & 0.001006 & 0.000146 & 0.000328 \\
\cline { 2 - 5 } & IPv6 & 0.001499 & 0.000135 & 0.000374 \\
\hline
\end{tabular}

Table 2: Results of Simulation.

\section{CONCLUSION}

This paper evaluated the performance of IPV4 and IPV6 over MPLS. The simulation results show that the IPv6 over MPLS network has higher throughput and link utilization than IPv4 over MPLS network. The traffic engineering used in MPLS keeps more packets in the network and with the help of it there is less packet drop. Besides, MPLS traffic trunks have been implemented which plays a wide role in increasing the link utilization.

It was also observed that the delay in case of IPv6 over MPLS network is higher than that of IPv4 over MPLS network. Furthermore, it can be said that the voice jitter in the IPv6 over MPLS network is higher than that of the IPv4 over MPLS network. Since delay is more in IPv6 over MPLS therefore jitter is also higher in this network. Finally, the simulation results shows that the IPv6 over MPLS network performs better than IPv4 over MPLS network in terms of throughput and link utilization while the IPv4 over MPLS has slightly lesser delay and jitter than IPv6 over MPLS.

\section{REFERENCES}

[1] Rozita Yunos, Noorhayati Mohamed Noor, Siti Arpah Ahmad, "Performance Evaluation between IPv4 and IPv6 on MPLS Linux Platform" Faculty of 
Computer and Mathematical Sciences, University of Technology-MARA.

[2] R. Ioan, "An Empirical Analysis of Internet Protocol version 6 (IPv6), Msc Thesis", Wayne State University, Detroit, Michigan, 2002.

[3] J. Barakovic, H. Bajric and A. Husic, "QoS Design Issues and Traffic Engineering in Next Generation IP/MPLS Network", 9th International Conference on Telecommunications (ConTEL 2007), 2007, pp. 203209.

[4] E. Rosen, A. Viswanathan, and R. Callon, RFC 3031: Multiprotocol Label Switching Architecture, (Proposed standard), 2001.

[5] Bernard Fortz, Jennifer Rexford, Mikkel Thorup "Traffic engineering with traditional IP routing protocols", IEEE Communications Magazine-2002.

[6] Mahesh Kr. Porwal., Anjulata Yadav., S. V. Charhate, "Traffic Analysis of MPLS and Non MPLS Network including MPLS Signalling Protocols and Traffic distribution in OSPF and MPLS," International Conference on Emerging Trends in Engineering and Technology, ICETET, July 2008.
[7] Nader F.Mir., Albert Chien, "Simulation of Voice over MPLS communications Networks," IEEE ICSS02 , conference.

[8] Md. Tariq Aziz et al. "Effect Of Packet Delay Variation On Video/Voice Over Diffserv-MPLS in IPV4/IPV6 Networks", International Journal of Distributed and Parallel Systems (IJDPS) Vol.3, No.1, January 2012.

[9] Luc De Ghein, CCIE No. 1897 "MPLS Fundamentals".

[10] O. Akinsipe et al. "Comparison of IP, MPLS and MPLS RSVP-TE Networks using OPNET", International Journal of Computer Applications (0975 - 8887) Volume 58- No.2, November 2012.

[11] X. Xiao et al. "Traffic Engineering with MPLS in the Internet" Global Center Inc. and Michigan State University, USA, vol. 14, pp. 28-33, Mar. 2000.

[12] A. Ghanwani et al. "Traffic Engineering Standards in IP Networks Using MPLS" IEEE Communication Magazine Dec. 1999. 